\title{
The growth dynamics of German business firms
}

\author{
Johannes Voit ${ }^{1}$ \\ Theoretische Physik 1, Universität Bayreuth, D-95440 Bayreuth (Germany), and \\ Fakultät für Physik, Albert-Ludwigs-Universität, D-79104 Freiburg (Germany)
}

\begin{abstract}
We determine the distribution of size and growthrates of German business firms in 1987-1997. We find a log-normal size distribution. The distribution of growth rates has fat tails. It can be fitted to an exponential in a narrow central region and is dominated by finite-sample-size effects far in its wings. We study the dependence of the growth rate distribution on firm size: depending on procedures, we find almost no dependence when the center of the distribution is considered or, similar to previous work, a power-law when the wings are weighted more strongly. Correlations in the growth of different firms are essentially random. We determine the annual growth of the entire economy, and successfully correlate it with a standard economic indicator of business cycles in Germany. We emphasize possible problems related to the finite number of firms comprised in our database and its short extension in time.
\end{abstract}

Key words: Company sizes; Firm growth; Log-normal, exponential, fat-tailed distributions; Business cycles; Economic indicators.

\section{Motivation, important problems}

To understand the dynamics of economic growth, and its underlying mechanisms, is important for society. Important issues are, among others, the influence and control of economic growth by government policy (e.g. taxes, employment conditions, or monetary policy); industrial concentration and antitrust policy, and its influence on competition and economic growth; changes in the structure of economies brought about by the globalization of economic activities, and their consequences. Also, it is unclear if firms which are part of the

1 e-mail: johannes.voit@uni-bayreuth.de, web: http://www.phy.uni-bayreuth.de/ btp314

Preprint submitted to Elsevier Preprint 26 October 2018 
"New Economy" of high-technology sectors grow according to the same rules as the firms of the "Old Economy", and if the same macroeconomic indicators should be used, e.g., to measure the business cycles of both economies.

Surprisingly, it turns out, however, that the dynamics of economies is not well understood, both empirically and with respect to its underlying mechanisms. "Unfortunately, as is obvious from reading the newspapers, the theory of macroeconomics is not a settled field. There is much controversy among economists what is a useful basic approach as well as about the detailed analyses of particular economic events and policy proposals" [1]. Economic growth, or the concentration of firms in an industry are often measured by some indicators, and the underpinning of these indicators by statistical data on an economy sometimes remains unclear. Quite generally, economic theories often focus on mean values, trends, macrovariables $[1,2]$.

Firm sizes in classical theory would be determined by the long-run cost curve: the cost of output has a U-shaped dependence on the amount of output, and is minimal at a specific scale. Firms therefore would grow or shrink under the influence of competition in the market, in order to attain this optimal size for maximal profits, and an equilibrium would establish at that size. The question, of course, is if and to what extent, the distributions of firms in an industry, or an entire economy, are consistent with such arguments and market mechanisms. It turns out, however, that both the arguments about, and even more so the evidences for, such "economies of scale" are controversial [3].

A different approach is taken by stochastic growth models [3] which attempt to describe the distribution of firm sizes in an economy, and relate details of these distributions to elements of the growth dynamics. The first such model was formulated by Gibrat [4] who postulated that the relative rate of growth of a company is independent of its size. Assuming further that the growth rates are uncorrelated in time and that firms grow independently of each other, he arrived at a description in terms of a multiplicative stochastic process leading to a log-normal distribution of firm sizes which was verified by his observations. Later work by economists also discussed other distribution functions involving power laws, but the evidence for or against a specific class of distributions has remained controversial [3]. Moreover, the discussion was strongly focussed on the shape of the size distribution and a correlation of this distribution with other properties of the sample was rarely attempted.

In the past few years, physicists have become interested in of economic problems, mostly financial markets [5]. It is tempting therefore to consider the dynamics of economic growth from a physicist's perspective. Growth processes are routinely investigated and modelled in Statistical Physics [6]. Examples are diffusion limited aggregation, or the Eden model, which may approximately describe the growth of crystals or tumors, respectively. Here, the identification 
of a specific growth mechanism is based on the form of the resulting object, i.e. on the correlation of its size (volume or mass) with its local fluctuations. This brings up the question: What can be learned from the study of fluctuations in economic growth?

This question is not new, and some papers have adressed growth properties of industries and economies [7-10]. Amaral et al., and Lee et al., found that (i) the size distribution of US firms and of the gross domestic product of 152 countries approximately follow log-normal distributions; (ii) the distribution of growth rates conditioned on the size of the firms (countries) are exponential; (iii) the standard deviation of this exponetial distribution depends on size as $\sigma \sim S^{-\beta}$ with $\beta \approx 0.16$ both for the US firms and for the countries. These findings were suggested to be universal, and indicative of the growth dynamics of systems with complex internal structures [7,8]. Takayasu and Okuyama [9] performed a similar analysis for different countries. They found that the shape of the firm size distribution depended on the country (power-law for the US, more exponential for France, Japan, and Italy) but obtained growth properties consistent with (ii) and (iii) above. Finally, Ramsden and Kiss-Haypál established the rank-size relation (Zipf analysis) for firms in the economies of 20 countries in 1994 [11]. Unless there is clustering at certain sizes, this procedure is rather equivalent to analyzing the cumulative distribution function. They found significant differences among the countries in their fits.

Interpretations were proposed in terms of systems with complex internal structure but no competition between firms $[7,8]$, competition of structureless firms [9], or thermodynamics [11]. The simulation of these models could reproduce essential features of the empirical data in all cases.

Important questions remain. One of the most immediate is about universality. How universal are the statistical properties of economies? Another problem is the correlation of a statistical analysis with economic indicators. To better understand these issues, we perform an analysis of the growth properties of German business firms.

\section{Method of present work}

We investigate two database of German business firms: (i) Datastream provides a data base with 570 stock companies over the 11 years 1987-1997; (ii) the Hoppenstedt data base contains about 6500 firms over 20 years. The investigation of this base is not completed at the time of writing so that only results based on the more limited database (i) will be presented.

We use the annual sales as an indicator of the size of a firm. Earlier work for US 
firms has shown, however, that other indicators such as assets or the number of employees, give similar results [12]. Due to the small sample size, we do not attempt to consider different industries. Earlier work for other countries shows that the results do not depend on the type of industry within a specific country $[9,12]$. Finally, we discard all firms with incomplete data from our sample. The structure of the sample suggests that in most cases, data are just "missing" randomly. However, we eliminate in this way also effects of mergers and acquisitions, as well as newly founded firms and firms going bankrupt.

Our data base finally contains 405 companies with sales data for the 11 years 1987-1997. Their sizes will be denoted by $S_{j}\left(t_{i}\right), j=1, \ldots, 405, i=$ $1987, \ldots, 1997$. One source of concern is the small size of this sample. Is it representative for the German economy? We attempt a preliminary answer by comparing to a standard indicator of economic growth below. More definite statements, however, will have to be based on studies using the bigger data base (ii), to be published elsewhere. Apart the limited number of companies sampled, we will point out repeatedly the problems associated with the finite extent in time, of our data base, and the limitations they imply for interpretation. Notice that many other studies suffer from similar limitations.

\section{Firm size distributions, annual growth}

Figure 1 shows the firm size distribution of the entire database $P\left[S_{j}\left(t_{i}\right)\right]$, i.e. all years 1987-1997 mixed. Similar results are obtained, though with less good

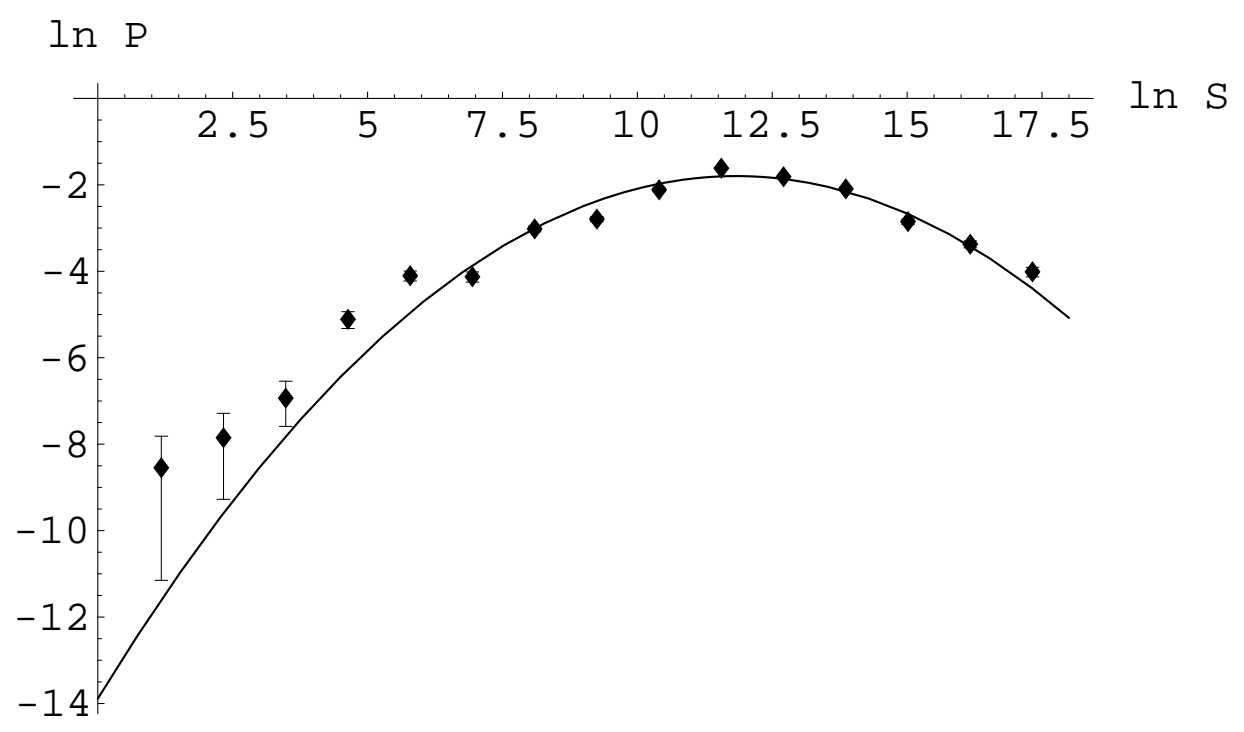

Fig. 1. Size distribution of German business firms. Dots: empirical data, line: fit to a log-normal distribution, parameters $\langle\ln (S)\rangle=11.8, \exp \langle\ln (S)\rangle=137 \times 10^{6} \mathrm{DM}$, standard deviation $=2.4$. 
statistics, when individual years are analyzed. The size distribution of German business firms indeed is approximately log-normal, in agreement with Gibrat's observations [4] and the US firms [7]. We note, however, that there is a sharp cutoff at big company sizes, and an excess of weight on the small-size wing of the distribution. These features are observed systematically with a one-year resolution, too. The cutoff at big sizes is consistent with similar observations for Japan, France, and Italy [9] but the data presentation in that work does not allow to draw conclusions on the small-size limit. We have not checked fits to alternative distribution functions.

From the firm sizes $S_{j}\left(t_{i}\right)$, annual growth rates $r_{j}\left(t_{i}\right)$ are derived as $r_{j}\left(t_{i}\right)=$ $\ln \left[S_{j}\left(t_{i}\right) / S_{j}\left(t_{i-1}\right)\right]$. The mean of the distribution of the annual growth rates gives the growth of the entire economy (to the extent that our limited database gives a faithful representation), and is shown in Figure 2. Despite the scatter, one can notice an apparently systematic variation of the economic conditions in Germany: there is a boom period in the late 80's and early 90's, slowing down and even recessing during the mid 90's and a restart of positive growth at the end of the sampling period of our database. While for residents of Germany this pattern will remind, and correlate with, the media reports on the economic conditions of the country, it is necessary to compare with established indicators of business cycles and economic trends, in order to assess the relevance of our analysis.

One such indicator for business cycles is provided by the (percentage) use of production capacity of German business firms [2]. Data for the manufactoring sector are reported annually by the Advisory Panel to the German Federal Government on the Economic Conditions, and shown in Figure 3 for the same period as our database. They show a pattern similar to the annual growth rate derived from our statistical analysis. This suggests that our data base

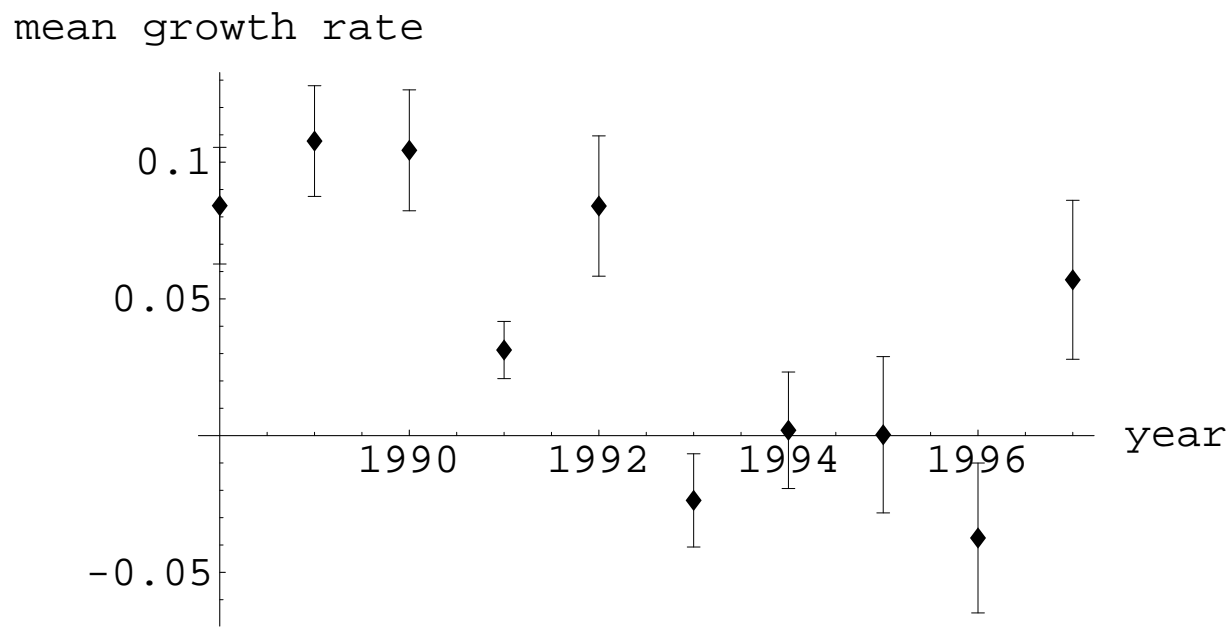

Fig. 2. Mean growth rate of the German economy as sampled by our database in 1987-1997. 


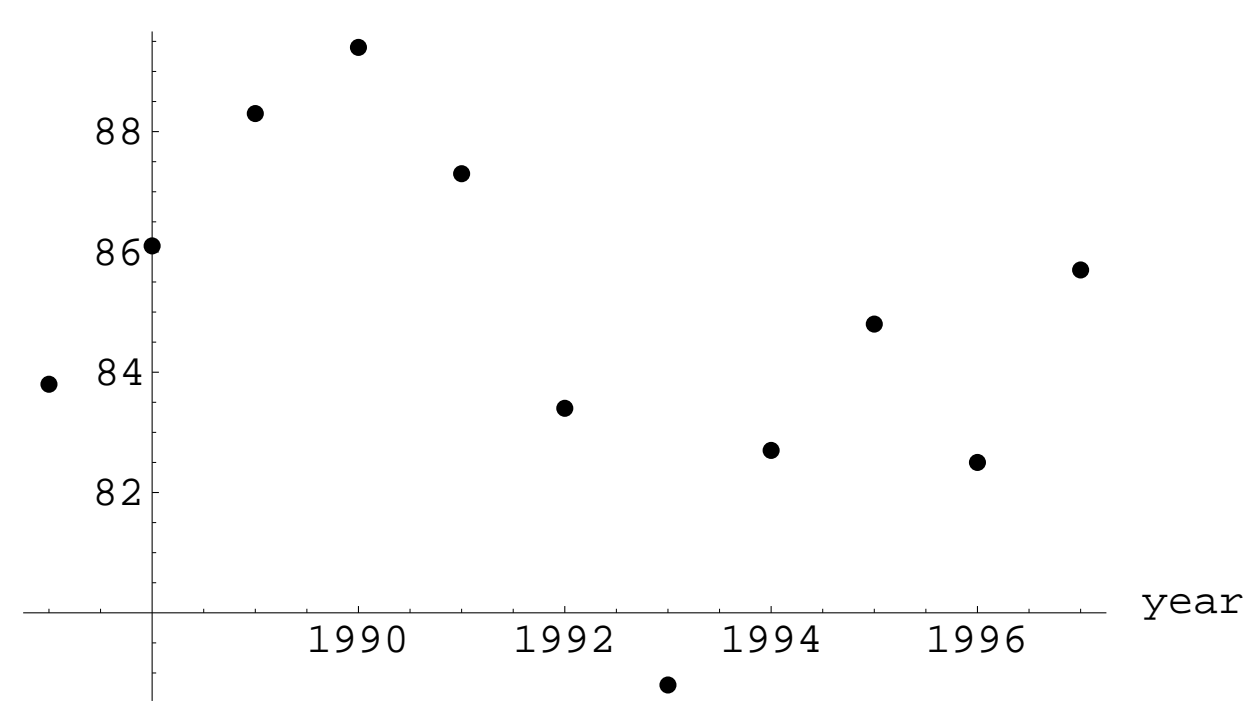

Fig. 3. Percentage use of production capacity of German manufactoring sector in 1987-1997.

may indeed provide a good representation of the German economy, despite its limited size; conversely, the agreement shows that the use of such coarsegrained indicators can be backed by a more "microscopic" statistical analysis. A priori, it is not obvious if this quantity, or rather its annual change should be compared to the mean growth rates shown in Figure 2. Its annual change, however, correlates less well with our analysis. We therefore conclude that the use of production capacity itself is the appropriate indicator.

\section{Growth rate distributions}

We now turn to the distributions of the firm growth rates. Figure 4 shows the distribution of the fluctuations in the growth rates (i.e. after the subtraction of the mean $\left.\left\langle r_{j}\left(t_{i}\right)\right\rangle_{i, j}\right)$, for the entire database, all years mixed. Apart the less good statistics, however, the distributions time-resolved on a one-year scale, show the same behavior. It is clear that the raw data have a fat-tailed distribution, far from both the normal distribution associated with a simple multiplicative stochastic process, and from the exponential distribution which has been found in earlier work [7-10]. At present, we have not attempted to fit the entire distribution to a particular form. We rather analyze separately the center and the wings of the distribution.

Figure 5 shows the distribution of the growthrates of firms with $-0.55 \leq$ $r_{j}\left(t_{i}\right)-\langle r\rangle \leq 0.45$. In this range, a successful fit to an exponential distribution is possible indeed (standard deviation 0.12). Hence we recover the exponential growth rate distributions found by Amaral et al., [7] and Takayasu and 
Okuyama [9], albeit only for a very narrow range of growth rates. The wings of the probability distributions are dominated by effects of finite sample size. The horizontal line in Figure 6, where the dots are the data shown in Figure 4 , represents the lower limit on counting, one count per bin. In the center of the figure, the exponential fit function derived in Figure 5 is superposed on the original data. Again, all features are recovered in time-resolved data on a one-year scale, with less good statistics.

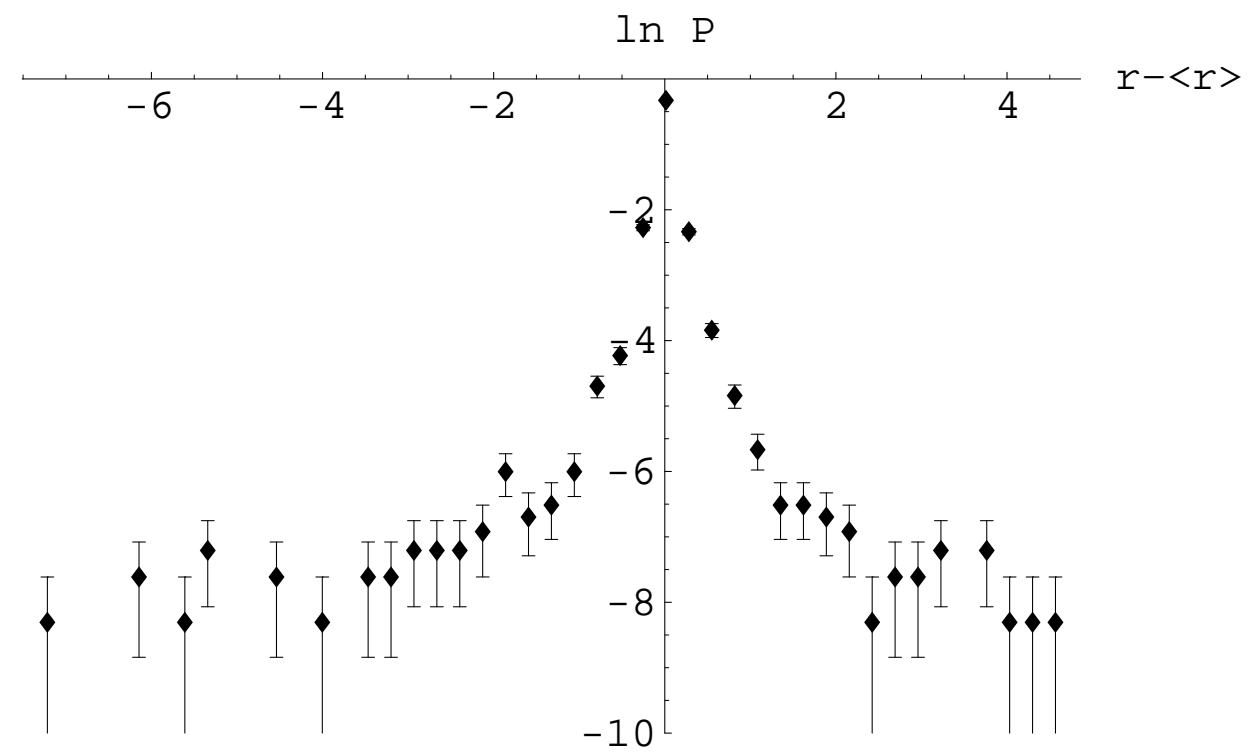

Fig. 4. Distribution of growth rates of German business firms with respect to their mean in 1987-1997.

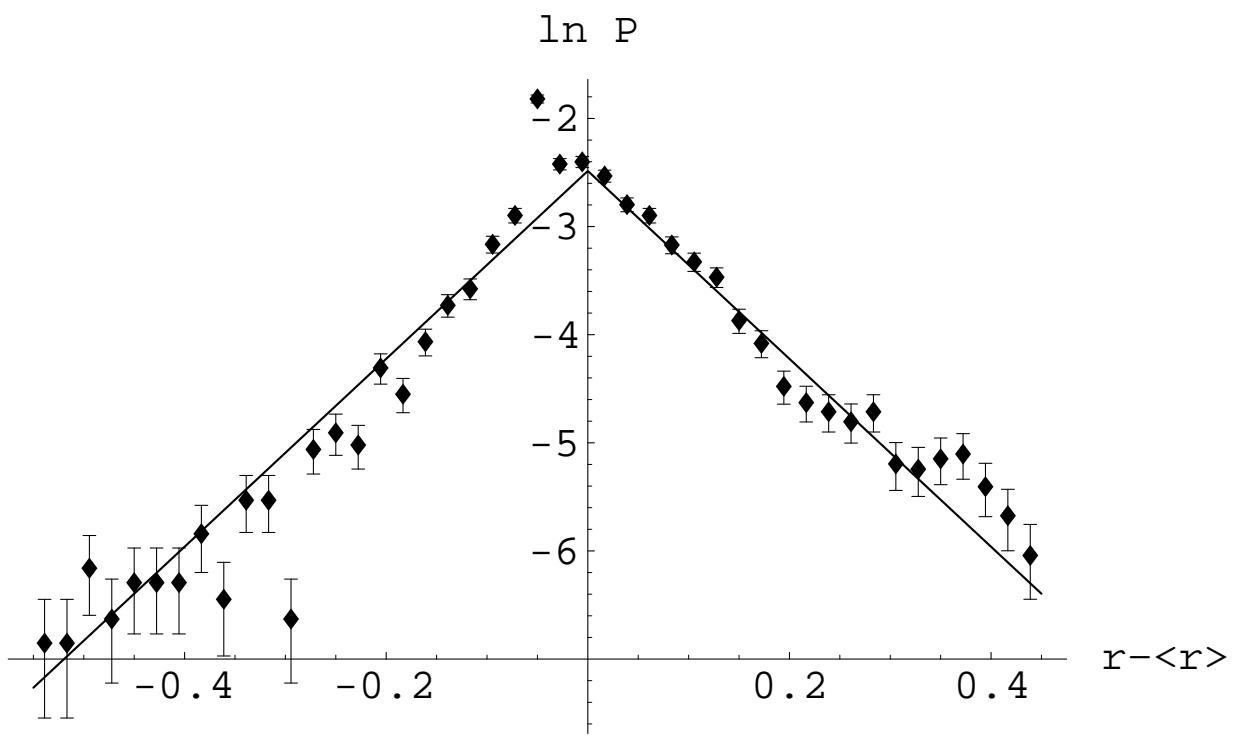

Fig. 5. Central part of growth rate distribution of German business firms in 1987-1997, and fit to an exponential distribution (solid line). 3788 out of 4050 data have been used in this analysis. 


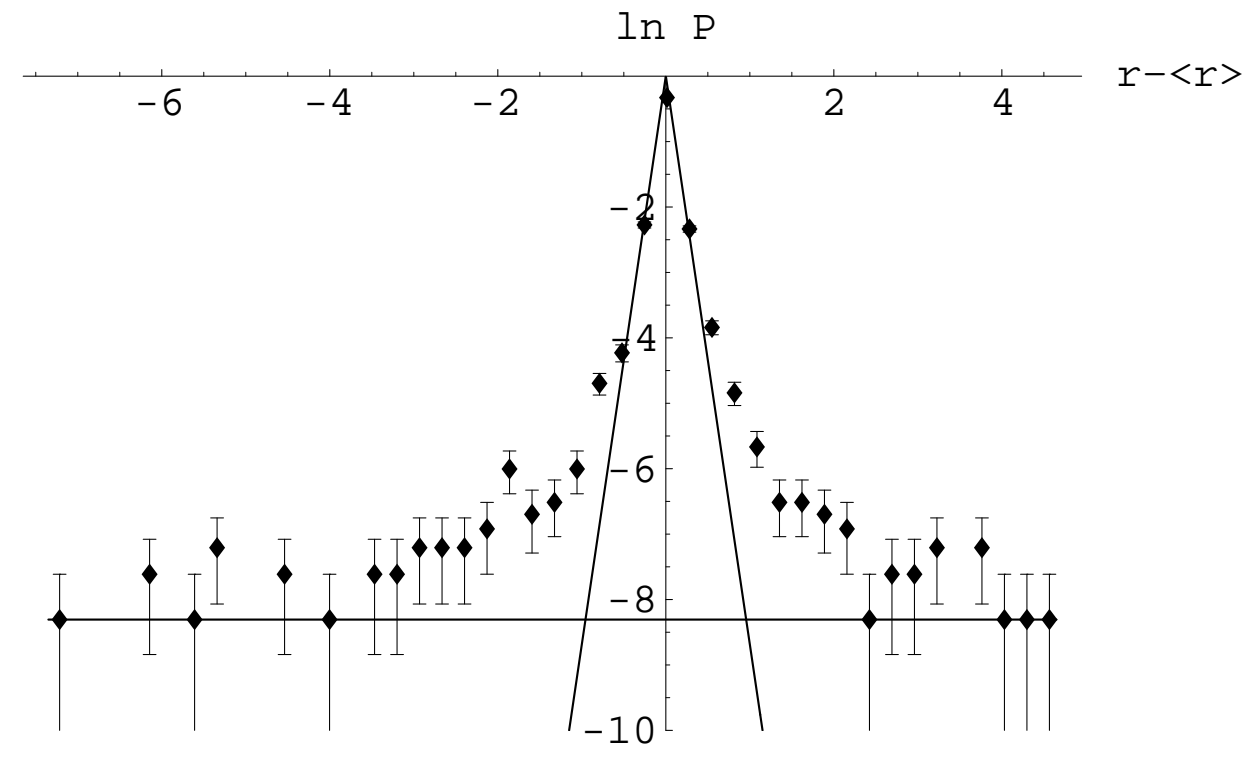

Fig. 6. Superposition of growth rate distribution of German business firms with central fit to an exponential distribution (tent-shaped solid line) and lower-finite size cutoff. The horizontal line corresponds to one count per bin.

Some observations, however, make us hesitate to endorse an exponential growth rate distribution at the present level of data analysis without reservations. They are related to the extremely reduced range over which the exponential fit can be performed. Counting rates are still rather high (50-200 firms per bin) in the range where deviations from exponential behavior are clearly marked already, and the deviation of the data from the exponential fit in the center is much larger than the error bars. The parameters derived from an exponential fit depend on the range of data used, and do not converge well as this range is reduced. Finally, the shape of our bare growth rate distribution, Figure 4, is very different from those found in other work $[7,9]$, and more reminiscent of work on stock exchange crashes which have been claimed to be outliers with respect to a presumed exponential distribution [14].

Earlier work made the interesting observation that both for firms, and for the gross domestic product of countries, the width $\sigma$ of the exponential growth rate distribution depends on the sizes of the firms, resp. countries, when the data set is binned according to firm or country size [7-9]. A power law dependence $\sigma \sim S^{-\beta}$ was found with a rather universal exponent $\beta \approx 0.16$. Figure 7 shows the corresponding result for German business firms. To generate this figure, we divided our firms into ten bins corresponding to the $n / 10$-quantiles of the sample. We then determined, the distribution of growth rates for each bin, and performed a fit to the center of the distributions as explained above. The resulting widths $\sigma$ are plotted vs. the average firm size in the respective bin, in Figure 7. There is a large scatter of points in the $\sigma-S$ plane, and no clear dependence of width on size emerges. If we - reluctantly - attempt a power law fit, we find an exponent $\beta=0.037 \pm 0.006$, essentially zero. If, 
instead, we use the standard deviation $\Sigma$ of the entire sample in each bin, we do recover the power law $\Sigma \sim S^{-\beta}$ with an exponent $\beta=0.19 \pm 0.02$, Figure 8 . This power law dependence essentially agrees with the earlier results [7-9], and supports the claims of universality made there. Notice, however, that this analysis more strongly reflects the variations in the wings of the growth rate distribution which may deviate from the exponential statistics discussed above, and which may suffer from severe finite sample-size effects.

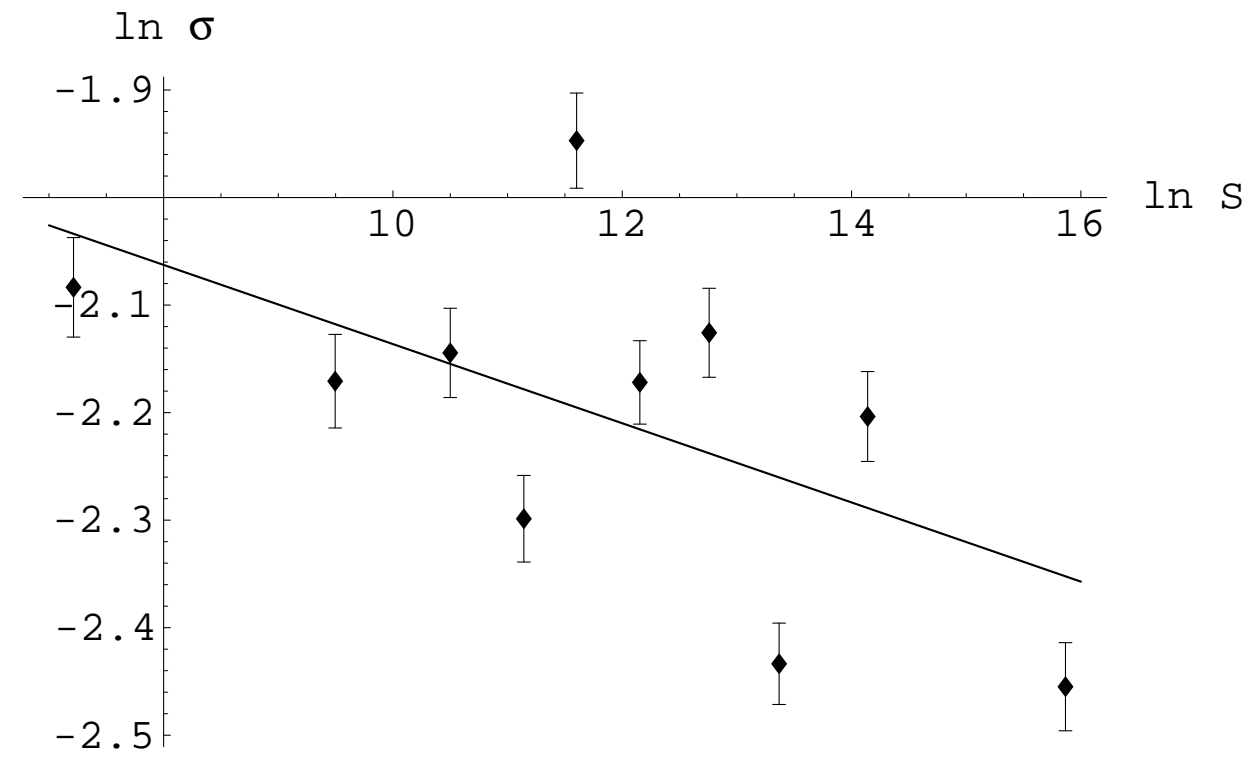

Fig. 7. Dependence of the width $\sigma$ of the exponential distribution fitting the center of $P(r-\langle r\rangle)$ vs. firm size. No clear dependence emerges. The solid line is the "best" fit to a power law $\sigma \sim S^{-\beta}$ with an exponent $\beta=0.037$.

$\ln \Sigma$

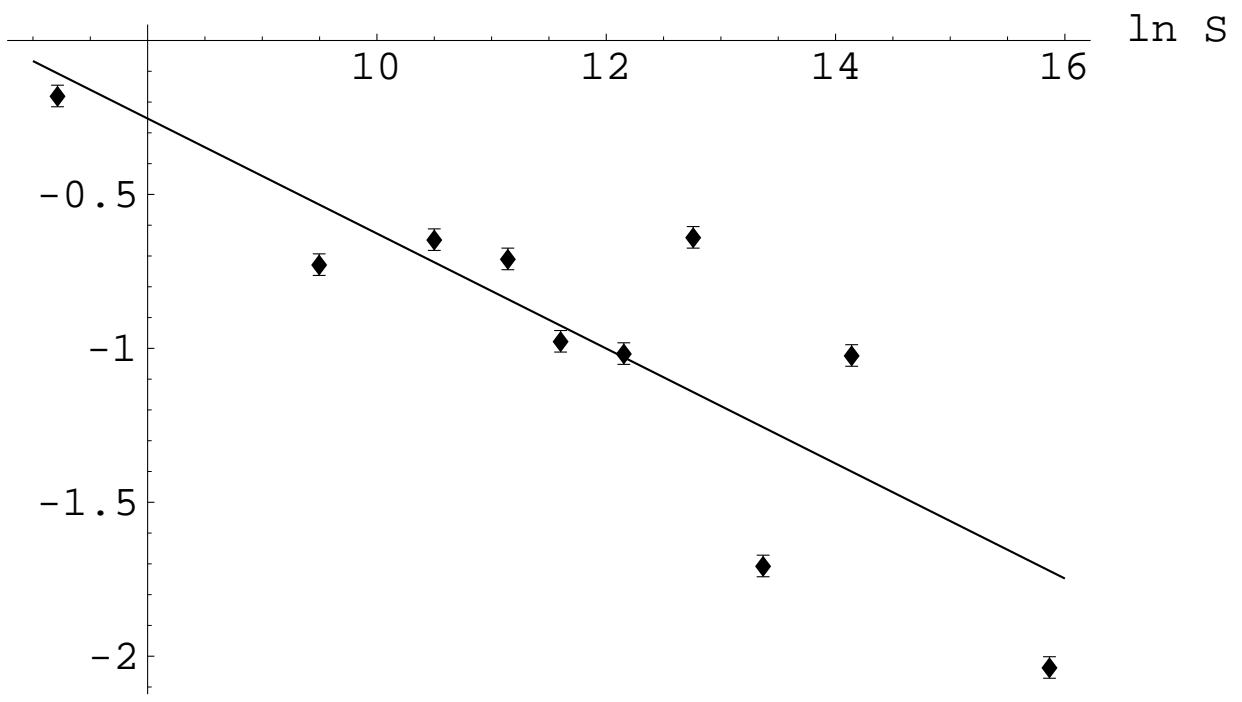

Fig. 8. Dependence of the standard deviation $\ln \Sigma$ of the individual bins on firm size. The solid line is a fit to a power law $\Sigma \sim S^{-\beta}$ with $\beta=0.19$. 
In the future, it will be important to understand the consequences of the dependence of the exponent $\beta$ on the sampling procedure.

It will also be important to study the reasons for the serious discrepancies between fitted laws and empirical data, in particular in Figures 7 and 8. In all figures, error bars are for one standard deviation. The number of data points deviating from the fitted laws in general is rather larger than $32 \%$. Similar conclusions would be reached at the $95 \%$ confidence level. It is not clear that going to larger samples would improve the situation: quite generally, the error bars will decrease with sample size. Also, the apparently random scatter of the data points around the fitted lines in Figs. 7 and 8 leaves little room for an improvement in the specification of the theory. However, there is the possibility that one of the assumptions underlying the error analysis is not satisfied: randomness of the data, i.e. growth rates. If the growth rates are strongly correlated, the number of independent entries in each of the histogram bins will be reduced significantly, and the error bars will be correspondingly increased.

\section{Correlations in firm growth}

Many models describing the observed firm size distributions, and those describing the exponential growth rate distributions [7], assume an uncorrelated growth of firms both in time, and across the economy. This assumption can be checked, in principle, from our data set. From an economic point of view, an assumption of uncorrelated firm growth may appear questionable, a priori. One the one hand, when a national economy evolves significantly and nonrandomly, this evolution should be visible in the growth dynamics of its members, and is likely caused by correlations of the individual growth processes. In periods where people buy more (less) cars, the sales of Daimler-Chrysler, Volkswagen, and BMW will increase (decrease) while changes in market share of the individual companies operate on much smaller scales. On the other hand, correlations between car producers and, e.g., pharmaceutical companies are less obvious and may even be negative.

We have computed the covariance matrix of the 405 firms of our database, and show results in Figures 9 and 10. For reasons of clarity, Figure 9 displays only the upper left $40 \times 40$ entries of the entire covariance matrix. Figure 10 shows a cut through the covariance matrix at row 199. Both figures apparently indicate that there are significant correlations in the growth of the firms [uncorrelated growth would lead to $\operatorname{cov}\left(S_{i}, S_{j}\right)=\delta_{i, j}$, and that these correlations are essentially random. Recent studies of financial markets indicate that

correlations between stock prices also have a strong random component [15]. There, about $94 \%$ of the correlations are consistent with pure randomness, 
and only $6 \%$ deviate significantly from such a hypothesis. One might then ask: Are economies glassy systems?

While the idea of random correlations in firm growth dynamics may have some appeal (cf. above), we caution against premature conclusions in this direction. In fact, the apparent randomness, or at least part of it, may be due to the small temporal extension of our data set. Sufficiently long time series are needed to make significant cross-correlations between various variables emerge, and clearly distinguish them from uncorrelated random variables. Even a rather large number of uncorrelated random variables will show some correlations provided one does not look at too long a time series ("noise dressing"). This is demonstrated on a surrogate data set in Figure 11. In this data set, "time series" for 405 "firms" and lengths of 10, 100, and 1000 entries ("years") have been produced with a random number generator. While the covariance matrix for 10 "years" is essentially indistinguishable from Figs. 9 and 10, and correlations are still pretty strong for a 100 "years", it is only for 1000 "year" long time series that the statistical independence of the random numbers becomes apparent. The upper left $40 \times 40$ entries of the covariance matrix for this latter case are shown in Figure 11.

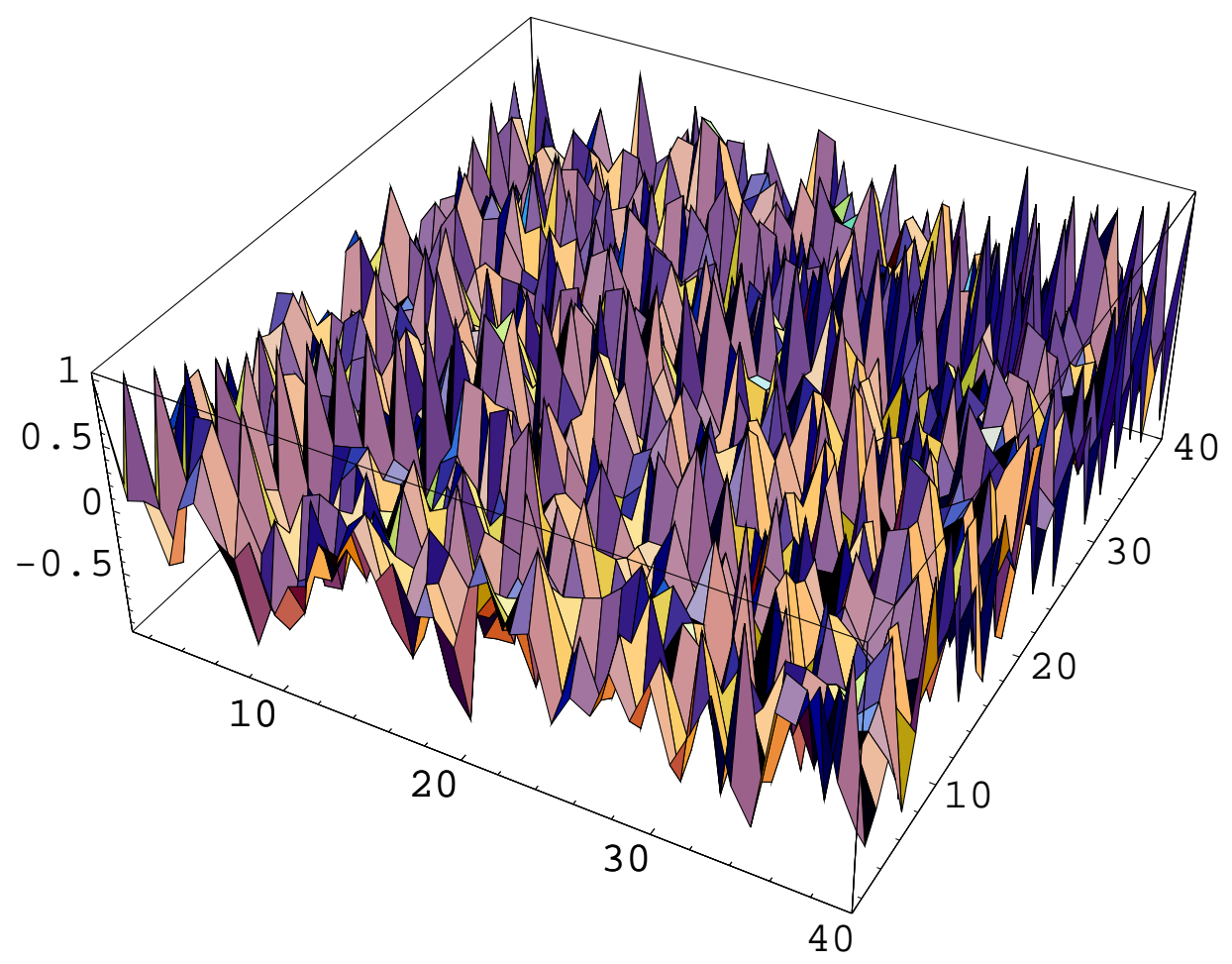

Fig. 9. Covariance matrix $\operatorname{cov}(i, j)$ of the growth rates of German business firms. For clarity, we only show the first $40 \times 40$ entries. 


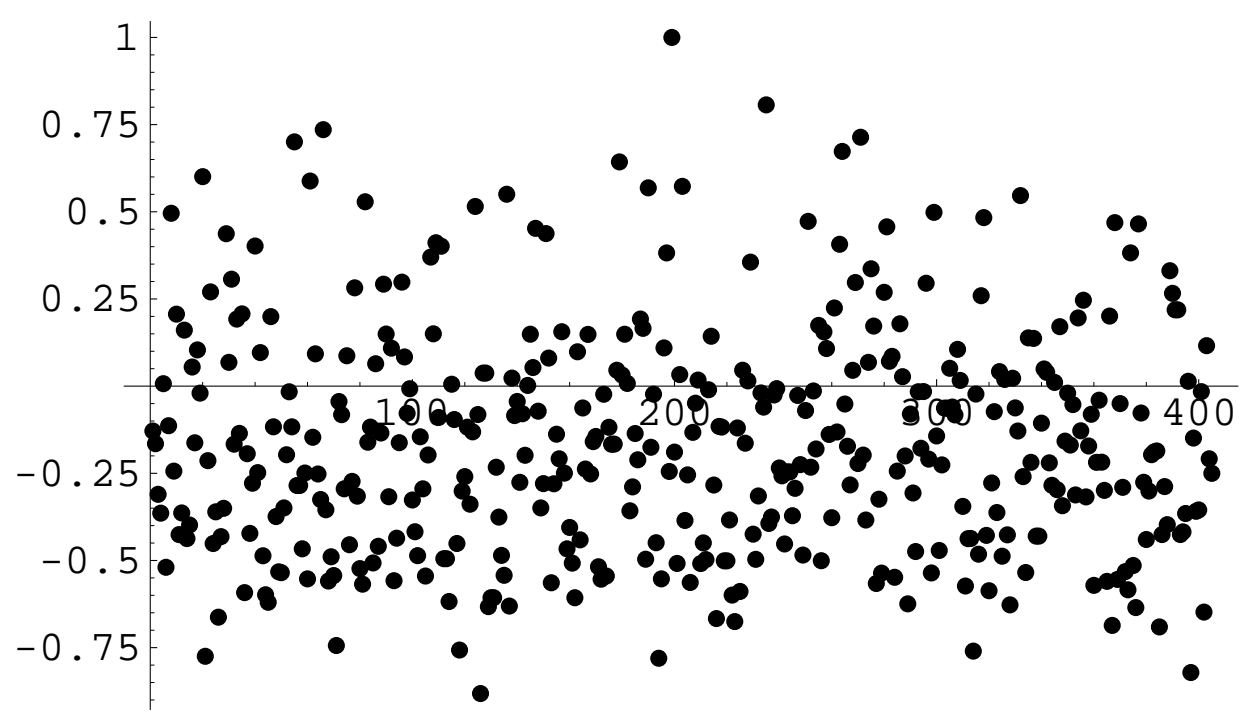

Fig. 10. Cut through the covariance matrix, $\operatorname{cov}(199, j)$, of German business firms.

6 Discussion, Open questions

The preceding analysis of the growth dynamics of German business firms shows notable similarities and differences to earlier work analyzing firms in other countries. The list of similarities include a log-normal size distribution, and

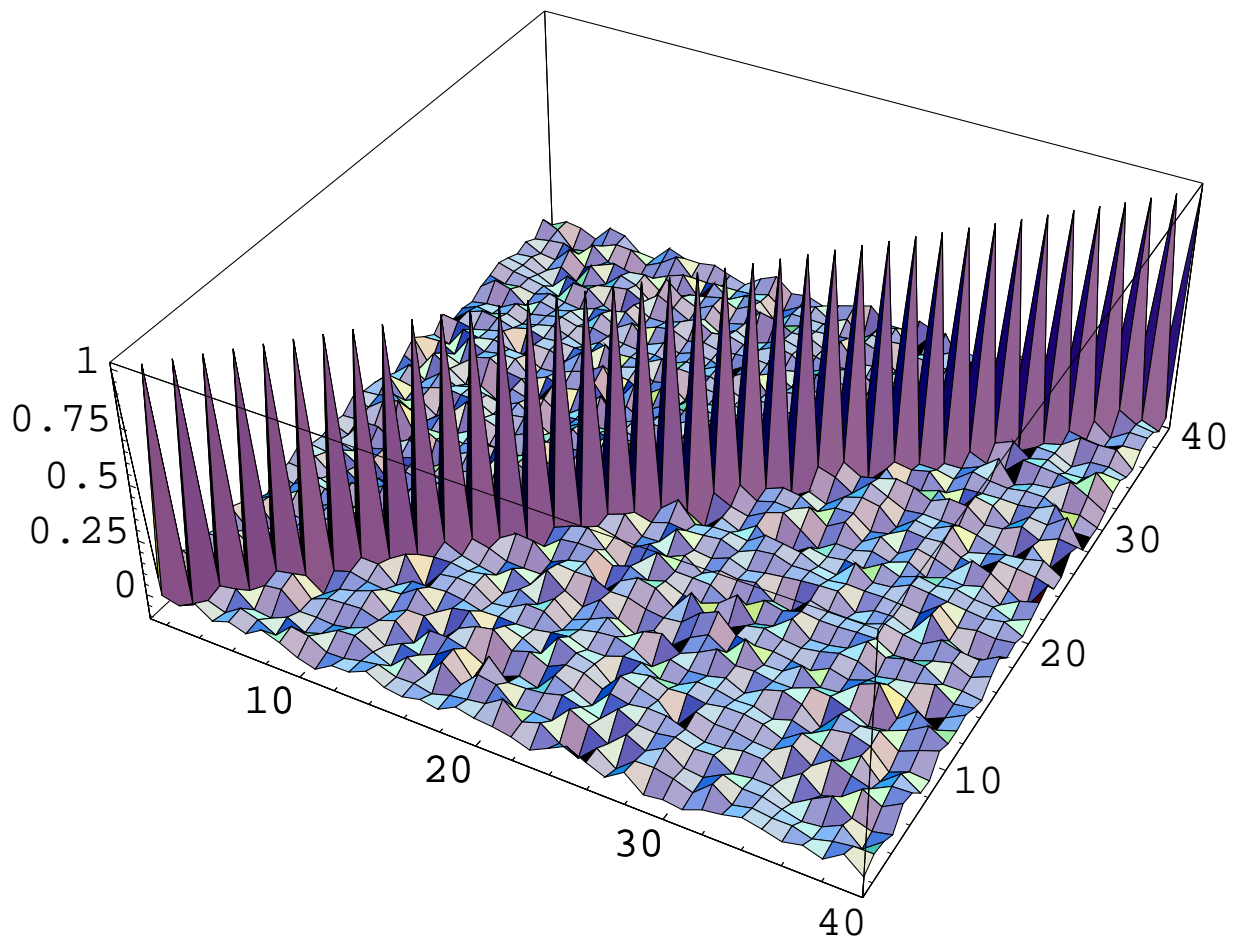

Fig. 11. Covariance of 405 series of random numbers with 1000 elements in each series. Only when the length of the series is comparable to the number of series, does the uncorrelated nature of the random numbers emerge. 
an exponential distribution of growth rates at least in the center of the distribution, albeit with some reservations with respect to the entire distribution. Differences are found (i) in certain systematic deviations from the log-normal distribution which is very well observed by US-firm or country data [7,8], and which may reflect specific features of the German economy, and (ii) in the dependence of the width of the growth rate distribution on firm sizes where no clear dependence emerges when the width of the exponential distribution in the center is used. However, we do observe a power law $\sigma \sim S^{-\beta}$ as found elsewhere [7-9] when the standard deviation of the entire sample in each bin is evaluated. It is not clear if the difference in the exponents $(\beta=0.19 \pm 0.02$ here vs. 0.16 elsewhere) is significant.

The cutoff in the firm size distribution for big firms could be related either to the size of Germany (the country can't support firms beyond a certain size) or, in a more global perspective, to the fact that the biggest multinational companies are based in the US. The excess of weight for small company sizes is much less clear and will require further investigation. It may possibly be related to a traditionally big number of small and medium-size enterprises in Germany ("Mittelständische Wirtschaft") which are well supported by politics, if they comprise a sufficient number of stock companies and therefore show up in our sample.

Several models have attempted to describe the size and growth rate distributions and the dependence of the latter on firm size, based on a distribution of minimal firm sizes necessary for survival in the different industries composing an economy [7], on the amount of competition in an economy and its control through, e.g., taxes [9], or on managerial culture (lean management vs. hierarchical and authoritarian) [16]. It will be interesting to interpret the size-independence of the growth rate distribution found here for the German economy, with specific features of these models, and to correlate these with independent economic information on German business firms. This is planned for future work. If successful, such a program could open the exciting possibility to correlate statistical properties of an economy with political, historical and cultural elements, and to closely monitor the changes brought about by both European integration and the worldwide globalization of the economy.

At the interface between statistics and economy, we have shown that the temporal evolution of the firms listed in our database tracks standard indicators of economic growth. On the other hand, we again caution against premature conclusions: Figure 12 shows the average growth of a surrogate economy (random numbers), and the differences to Figures 2 and 3 apparently are minor. The similarity of these figures makes us wonder to what extent the variations in the growth of the German economy are systematic, i.e. reveal business cycles, and how big a random component they contain. On what time scales does nonrandomness become important? Data for the growth rate of the gross national 


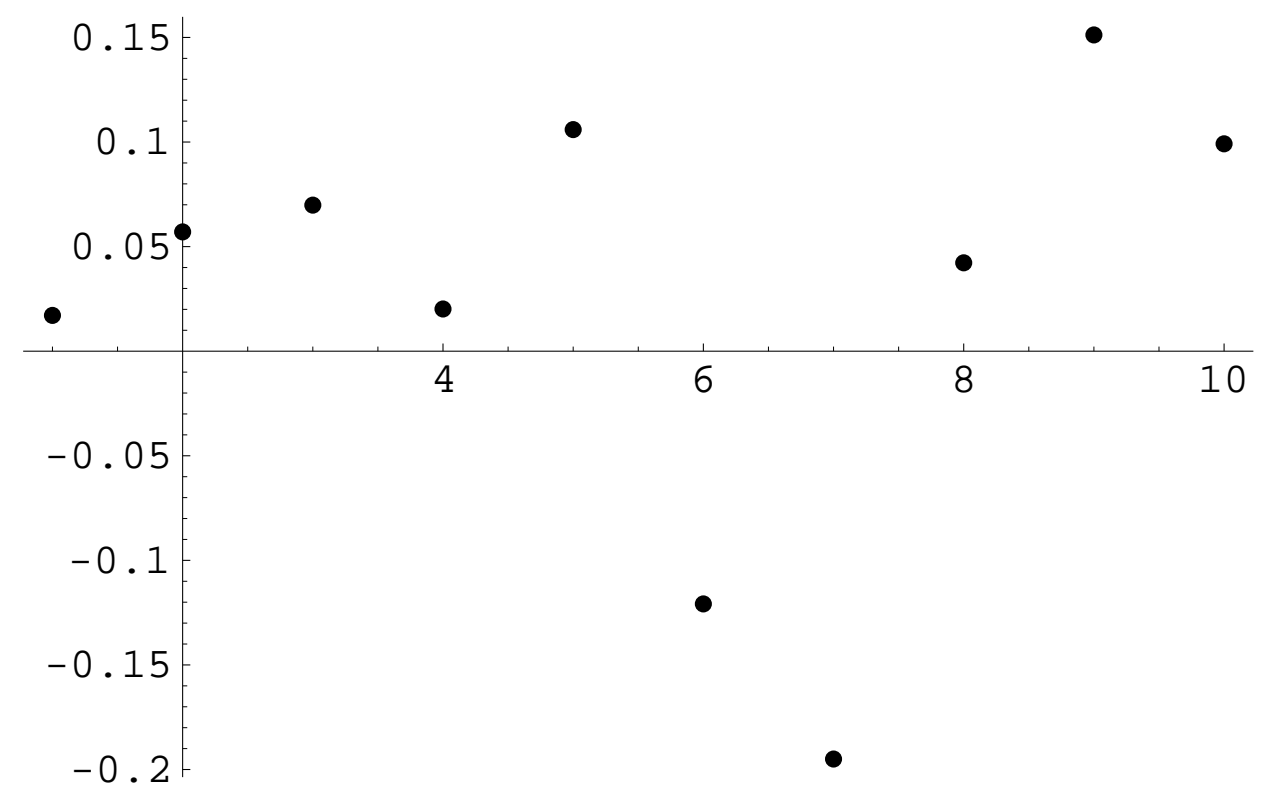

Fig. 12. "Annual growth" of a surrogate economy (random numbers). Systematic and random variations cannot be distinguished for few entries only.

product of the US 1870-1988 look random superposed on a small positive offset while those for Germany 1950-1992 show a more periodic structure [17]. This points to an important limitation of economic time series, compared to time series in physics, finance, medicine, etc., namely their short extension in time. Gross indicators are available over rather long times but detailed firm data have been collected rather recently only. Finite sample length effects are a serious problem, and may also have affected the analysis presented here.

Future work therefore should address temporal correlations, and the importance of business cycles. Some evidence in favor of temporal correlations has been gathered in the past [3]. It would also be of interest to investigate birth and death processes in the economy. Finally, it will be interesting to confront the rather pragmatic approach of a physicist used here, to the more elaborate tools used by econometricians such as hypothesis tests, etc. These issues will be taken up in future publications.

\section{Acknowledgements}

I am a Heisenberg fellow of Deutsche Forschungsgemeinschaft. Most of all, I would like to acknowledge advise from and discussions with Dirk Obert (Freiburg) who also provided the data used here. This contact has been instrumental in getting this work started. I also would like to acknowledge discussions with S. Hügle, C. Jelitto, C. Kreuter, V. Plerou, and H. E. Stanley. 


\section{References}

[1] R. J. Barro, Macroeconomics, 3rd. ed., J. Wiley \& Sons, Inc., New York (1990).

[2] G. Blümle and W. Patzig, Grundzüge der Makroökonomie, 3rd. ed., Rudolf Haufe Verlag, Freiburg (1993).

[3] Y. Ijiri and H. A. Simon, Skew Distributions and the Sizes of Business Firms, North-Holland Publ. Comp., Amsterdam (1977), and references therein.

[4] R. Gibrat, Les inégalités économiques, Sirey, Paris (1931).

[5] E.g., J.-P. Bouchaud and M. Potters, Théorie des Risques Financiers, AléaSaclay, Paris (1997); R. N. Mantegna and H. E. Stanley, An Introduction to Econophysics, Cambridge University Press, Cambridge (2000); J. Voit, The Statistical Mechanics of Capital Markets, Lecture notes, Universität Freiburg (1999), cf. http://www.phy.uni-bayreuth.de/〜btp314/lecnotes.html.

[6] On Growth and Form, ed. by H. E. Stanley and N. Ostrowski, Martinus Nijhoff Publishers, Boston (1986).

[7] L. A. N. Amaral, S. V. Buldyrev, S. Havlin, M. A. Salinger, and H. E. Stanley, Phys. Rev. Lett. 80, 1385 (1998).

[8] Y. Lee, L. A. N. Amaral, D. Canning, M. Meyer, and H. E. Stanley, Phys. Rev. Lett. 81, 3275 (1998).

[9] H. Takayasu and K. Okuyama, Fractals 6, 67 (1998).

[10] Some of this work is critically reviewed (in German) in D. Obert, Ch. Jelitto, S. Hügle and J. Voit, Phys. Bl. 55, 14 (February 1999).

[11] J. J. Ramsden and Gy. Kiss-Haypál, Physica A 277, 220 (2000).

[12] L. A. N. Amaral, S. B. Buldyrev, S. Havlin, H. Leschhorn, P. Maass, M. A. Salinger, H. E. Stanley, and M. H. R. Stanley, J. Phys. (Paris) I 7, 621 (1997).

[13] Jahresgutachten 1996/97 des Sachverständigenrates zur Begutachtung der gesamtwirtschaftlichen Entwicklung, Deutscher Bundestag, Drucksache 13 / 6200, Bonn (1996). Wirtschaftspolitik unter Reformdruck, Jahresgutachten 1999/2000 des Sachverständigenrates zur Begutachtung der gesamtwirtschaftlichen Entwicklung, Metzler-Poeschel, Stuttgart (1999).

[14] D. Sornette and A. Johansen, Eur. Phys. J. B 1, 141 (1998).

[15] L. Laloux, P. Cizeau, J.-P. Bouchaud, and M. Potters, Phys. Rev. Lett. 83, 1467 (1999); V. Plerou, P. Gopikrishnan, B. Rosenow, L. A. N. Amaral, and H. E. Stanley, Phys. Rev. Lett. 83, 1471 (1999).

[16] S. V. Buldyrev, L. A. N. Amaral, S. Havlin, H. Leschhorn, P. Maass, M. A. Salinger, H. E. Stanley, and M. H. R. Stanley, J. Phys. (Paris) I 7, 635 (1997).

[17] US: Figure 1.2 in Barro, [1]; Germany: plate 3, p. 499 in Blümle and Patzig, $[2]$. 